\title{
LEOMYOSARCOMA OF THE BRONCHUS: REPORT OF TWO CASES OF RESECTION WITH LONG-TERM FOLLOW-UP
}

\author{
Giuseppe Muscolino, MD, Amedeo V. Bedini, MD, and Paolo F. Buffa, MD, Milan, Italy
}

Primary pulmonary leiomyosarcomas include bronchial and parenchymal forms and are rare in surgical series. To our knowledge few cases have been reported in the Western literature $^{1-4}$ to date. We have performed more than 3000 lung resections at our department since 1990, and in this series we observed only two patients affected by primary leiomyosarcoma of the bronchus in 1992. These two underwent pulmonary resection with bronchoplasty and their case histories are reported herein.

\section{Clinical summaries}

PATIENT 1. A 69-year-old man had a dry cough of 3 to 4 months' duration and chest x-ray evidence of an abnormal perihilar shadow. Antimicrobial treatment had been administered for 1 month but had not modified this shadow, and he was referred to us. Thoracic computed tomographic scanning performed in our institute revealed a mass involving the right upper lobe and bronchus (Fig 1), and bronchoscopic examination showed that the latter was completely obstructed by a polypoid lesion protruding into the main stem bronchus. Examination of biopsy tissue revealed squamous metaplasia and chronic inflammation. Repeated bronchoscopic examina-

From the Department of Thoracic Surgery, National Cancer Institute, Milan, Italy.

Received for publication March 16, 1999; accepted for publication Jan 5, 2000

Address for reprints: Giuseppe Muscolino, MD, Galleria Strasburgo 1/2, 20122 Milano, Italy.

J Thorac Cardiovasc Surg 2000;119:853-4.

Copyright $\odot 2000$ by The American Association for Thoracic Surgery. $0022-5223 / 2000 \$ 12.00+0 \quad \mathbf{1 2 / 5 4} / \mathbf{1 0 5 4 5 3}$

doi:10.1067/mtc.2000.105453

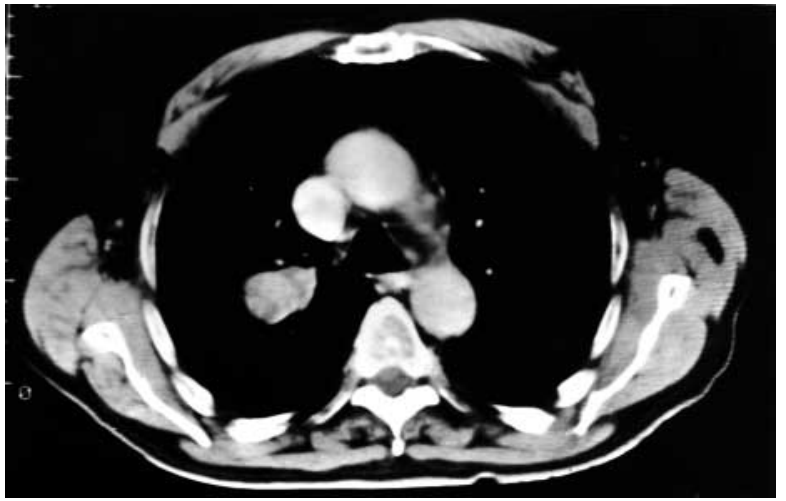

Fig 1. Thoracic computed tomographic scan of patient 1 .

tions confirmed the macroscopic appearance, and biopsy tissue revealed submucosal leiomyomatous cells. Upper lobectomy with wedge resection of the main stem bronchus was performed via an anterior thoracotomy. Anatomopathologic assessment showed a grade 1 leiomyosarcoma (diameters: $3.8 \times 4 \mathrm{~cm}$ ) arising from the lobar bronchial wall with no involvement of lung parenchyma.

PATIENT 2. A 62-year-old woman was referred to our department for bronchoscopic examination because of a 1- to 2month history of blood-stained sputum. The study revealed an $0.5 \mathrm{~cm}$ lesion of the carina and right main stem bronchus. Examination of biopsy tissue showed squamous metaplasia and bronchial chronic inflammation. The patient was treated with Nd:YAG laser therapy. Bronchoscopic reassessment after 2 months revealed recurrence and macroscopic features resembling those observed at initial assessment. Repeated 
biopsy and Nd:YAG laser treatment were performed by 1stage bronchoscopy, and grade 1 leiomyosarcoma was diagnosed. Carinal resection with upper right lobectomy was carried out via an anterior thoracotomy, such as we usually use for sleeve pneumonectomy. ${ }^{5}$ Bronchial reconstruction consisted of end-to-end anastomosis between the trachea and intermediate bronchus, with the medial wall of the bronchus being sutured to the medial wall of the left main stem bronchus. Microscopic assessment showed reparative processes in the carina and medial wall of the right main stem bronchus due to the Nd:YAG laser treatment.

Both patients had uneventful recoveries and neither received adjuvant treatment. Both are alive and disease-free. Duration of postoperative follow-up is 7 years for the man and 6.5 years for the woman.

Discussion. Primary pulmonary leiomyosarcoma is rare. The lung is the most common site of metastatic spread of tumors arising from the uterus, digestive tract, retroperitoneum, or deep soft tissue, and accurate assessment of these organs is required to rule out primary malignant disease in cases of solitary pulmonary leiomyosarcoma. ${ }^{4}$ Moreover, exhaustive evaluation of the lung and adrenal glands is mandatory, as these are the most frequent sites of metastatic spread from lung leiomyosarcoma. ${ }^{3}$

Pulmonary leiomyosarcoma can be subdivided into parenchymal and bronchial forms. The former can reach a considerable diameter without involving the bronchial wall ${ }^{6}$ and can remain symptom-free for a prolonged period. The bronchial form can be detected with a bronchoscope and is often associated with early cough and hemoptysis. These characteristic symptoms allow early diagnosis. Our patients had circumscribed tumors, and curative resection was achieved in both. In neither patient did the biopsy tissue obtained by the initial endoscopic examination lead to the final diagnosis. It showed submucosal leiomyomatous cells in case 1 and squamous metaplasia of the epithelium in case 2 because of the submucosal growth pattern of the tumor. In our patients the correct diagnosis was achieved only after specimens were obtained from deep mucosal layers. Both patients had favorable prognostic factors (ie, bronchial tumor location and low histologic grade) and were successfully treated with lung-sparing surgery and bronchoplasty. Radical and curative resection did not necessitate wide free margins, and the effective long-term control of the disease implies that these tumors are not very aggressive locally. Surgery appears to be the treatment of choice. We believe that conservative procedures should be used in bronchial leiomyosarcoma when feasible.

\section{REFERENCES}

1. Yacoubian H, Connolly JE, Wylie RH. Leiomyosarcoma of the lung. Ann Surg 1958;147:116-9.

2. Dyson BC, Trentalance AE. Resection of primary pulmonary sarcoma. J Thorac Cardiovasc Surg 1964;47:577-9.

3. Ramanathan T. Primary leiomyosarcoma of the lung. Thorax 1974;29:482-9.

4. Conte B, Leitner J. Leiomyosarcoma of the lung. J Thorac Cardiovasc Surg 1993;105:1119-20.

5. Muscolino G, Valente M, Ravasi G. Anterior thoracotomy for right pneumonectomy and carinal reconstruction in lung cancer. Eur J Cardiothorac Surg 1992;6:11-4.

6. Warren H, Bleck P, Kittle CF, Faber LP. Surgical management of pulmonary metastatic leiomyosarcoma with gross endobronchial extension. Ann Thorac Surg 1990;50:739-42. 